schlechterung während einer diagnostischen bronchoalveolären Lavage vorzubeugen [1]. Die vorliegende Studie geht noch einen Schritt weiter und liefert die wissenschaftliche Evidenz dafür, dass die Bronchoskopie unter NIV auch bei COPD-Patienten mit akuter respiratorischer Azidose unter schwierigen Bedingungen möglich und therapeutisch sinnvoll ist.

Bemerkenswert ist dabei insbesondere, dass die bronchoskopische Absaugung in allen Fällen erfolgreich und ohne Komplikationen möglich war, obwohl ausschließlich Patienten aufgenommen wurden, die neben der Sekretproblematik bereits eine Bewusstseinstrübung aufwiesen (KellyMatthay-Score 2-4).

Tatsächlich dürfte unter diesen Bedingungen zumindest bei einigen Klinikern mit geringer Erfahrung im Umgang mit der NIV die primäre Intubation die Therapie der ersten Wahl sein. Dieser Argumentation folgend wurden Patienten mit eingeschränkter Sensorik tatsächlich in den meisten randomisierten prospektiven Studien zur NIV bisher ausgeschlossen.

In diesem Zusammenhang ist darauf hinzuweisen, dass diese Studie in einem Zentrum mit hoher Expertise im Umgang mit der NIV und wohl auch mit der Bronchoskopie durchgeführt wurde. Die Ergebnisse dürften daher nicht ohne Weiteres auf alle Intensivstationen übertragbar sein. Die bronchoskopische Absaugung erfordert unter diesen Bedingungen neben technischem Geschick und Expertise eine zügige Untersuchung und ein engmaschiges Monitoring, um den Patienten in dieser ohnehin schon schwierigen Situation nicht weiter zu gefährden.

Aufgrund einiger Limitationen der Studie kann der Erfolg dieser Strategie noch nicht abschließend beurteilt werden. Es ist aber ein ermutigendes Ergebnis, dass in der NIVGruppe trotz des höheren Alters und der höheren Komorbidität signifikant weniger infektiöse Komplikationen beobachtet wurden, obgleich sich dies nicht in einer geringeren Klinikmortalität widerspiegelte. Letzteres muss in weiteren prospektiven kontrollierten Studien gezeigt werden.

\section{Literatur}

1. Antonelli $M$ et al. Noninvasive positive-pressure ventilation vs. conventional oxygen supplementation in hypoxemic patients undergoing diagnostic bronchoscopy. Chest 2002;121:1149-1154

\title{
Postoperative Pneumonien
}

\section{Kein erhöhtes Risiko durch Säureblocker}

Redelmeier DA et al. Postoperative pneumonia in elderly patients receiving acid suppressants: a retrospective cohort analysis. BMJ 2010;340:C2608

\section{Hintergrund und Fragestellung}

Eine Pneumonie ist eine häufige und schwerwiegende Komplikation elektiver chirurgischer Eingriffe. Nun wurde untersucht, ob Säureblocker mit einem erhöhten Risiko für postoperative Pneumonien assoziiert sind.

\section{Methodik}

In die retrospektive Kohortenanalyse wurden 593265 zu einer elektiven Operation aufgenommene Patienten über 65 Jahren eingeschlossen.

\section{Ergebnisse}

21\% der Patienten nahmen einen Säureblocker (am häufigsten Omeprazol oder Ranitidin). 6389 Patienten entwickelten eine postoperative Pneumonie. Bei Patienten, die Säureblocker einnahmen, war die Rate postoperativer Pneumonien (13 pro 1000$)$ signifikant höher als bei den anderen Patienten (10 pro 1000). Die Häufigkeit einer postopera- tiven Pneumonie nahm damit bei Einnahme von Säureblockern um 30\% zu (95\%-Konfidenzintervall [KI]: 1,23$1,38 ; \mathrm{p}<0,001)$. Allerdings wurde nach der Adjustierung für die Operationsdauer, das Operationsgebiet und andere Einflussgrößen keine Risikozunahme gefunden (Odds Ratio $=1,02 ; 95 \%-\mathrm{KI}$ : 0,96-1,09; $\mathrm{p}=0,48)$.

Erwartungsgemäß traten andere Faktoren als signifikante Prädiktoren einer postoperativen Pneumonie zutage. Sehr wichtig war die Art des chirurgischen Eingriffs: Das höchste Risiko fand sich bei thorakalen Eingriffen (adjustierte Odds Ratio $=2,72 ; 95 \%$-KI $=2,46-$ 3,00).

Weitere signifikante Risikofaktoren waren bestimmte vorbestehende Erkrankungen (z.B. COPD, Herzinsuffizienz, M. Parkinson), das Vorhandensein einer Magensonde und die Einnahme bestimmter Arzneimittel (z.B. Antipsychotika).

\section{Schlussfolgerung}

Säureblocker sind nicht mit einem erhöhten Risiko einer postoperativen Pneumonie bei älteren Patienten assoziiert.

\section{Kommentar}

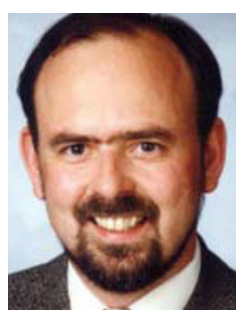

Prof. Dr. med. Klaus Mörike, Tübingen

Die wichtigste Limitation dieser Arbeit liegt darin, dass es sich nicht um eine randomisierte Studie handelt. Die Eliminierung aller Störeinflüsse ist damit nicht möglich. Hinzu kommt, dass negative Studien anderen Verzerrungen als Studien mit signifikanten Ergebnissen unterliegen können. Ein wesentlicher Vorteil dieser Studie ist die sehr große Patientenanzahl und die daraus resultierende hohe statistische Power.

Als Schlussfolgerung daraus, dass den Säureblockern offenbar nicht die Hauptsorge bezüglich der Entwicklung einer postoperativen Pneumonie gilt, sollte das Augenmerk auf andere Faktoren gerichtet werden. Diese könnten z.B. die Beendigung des Rauchens, die Optimierung der Ernährung, die Reduktion psychoaktiver Arzneimittel und ein frühzeitiges Absetzen von Magensonden beinhalten. 